\title{
PRESSURE AND TEMPERATURE DEPENDENCE OF THE PROTON AND DEUTERON SPIN-LATTICE RELAXATION TIMES IN LIQUID $\mathrm{H}_{2} \mathrm{~S}$ AND $\mathrm{D}_{2} \mathrm{~S}$
}

\author{
J. HAUER, E.W. LANG and H.-D. LÜDEMANN \\ Institut für Biophysik und Fhysikalische Biochemie, Universität Regensburg, D-8400 Regensburg, FRG
}

Received 4 May 1981

\begin{abstract}
The pressure and temperature dependence of the spin-lattice relaxation time $T_{1}$ for the protons in liquid $\mathrm{H}_{2} \mathrm{~S}$ and the deuterons in liquid $\mathrm{D}_{2} \mathrm{~S}$ have been measured between the melting-pressure-curve and $395 \mathrm{~K}$ at pressures up to $200 \mathrm{MPa}$. The different contributions to the experimental relaxation rate $T_{1}^{-1}$ could be separated with the extended $J$-diffusion model. The results are compared with analogous measurements made previously in liquid water and liquid ammonia in order to get some insight into the infiuence of hydrogen bonding interactions upon molecular motions in these liquids. From the pressure dependence of the isotherms an activation volume of $\Delta V^{\dagger}=2 \pm 0.5\left[\mathrm{~cm}^{3} / \mathrm{mol}^{\prime}\right.$ is derived for the rotational motion of the $\mathrm{H}_{2} \mathrm{~S}$ molecule, while the translational motion is characterized by a higher $\Delta V^{\star}=7 \pm 1\left[\mathrm{~cm}^{3} / \mathrm{mol}^{*}\right.$.
\end{abstract}

\section{Introduction}

The hydrides of nitrogen, oxygen and fluorine form liquids with rather unique structures. The physical properties of these liquids are explained by the ability of these hydrides to participate in hydrogen bonding. The physical anomalies caused by the formation of extended hydrogen bond networks are most pronounced in the case of liquid water [1]. Liquid hydrogen sulfide, the higher homologue of water, does not reveal any hydrogen bonding ability. The molecular interactions in hydrogen sulfide are dominated mainly by dipole- and dispersion forces $[2,3]$, so that it may be regarded as a simple dipolar, non-associated liquid. Its molecular geometry is similar to the geometry of the water molecule $[4,5]$ as is its dipole moment $\left(\mathrm{H}_{2} \mathrm{O}: 1.8 \times 10^{-18}\right.$ esu; $\mathrm{H}_{2} \mathrm{~S}: 1.1 \times$ $10^{-18}$ esu; $\left.\mathrm{NH}_{3}: 1.3 \times 10^{-18} \mathrm{esu}\right)$. Thus it may be hoped that comparison of the dynamic properties of these hydrides will yield some insight into the influence of hydrogen bonds upon the molecular motions in these liquids.
As is well known, NMR presents a suitable method for the study of molecular motions in liquids. Valuable information can be gained from the temperature and pressure dependence of spin-lattice relaxation times $T_{1}$. Previous NMR investigations of this substance have mainly been concerned with its properties in different solid phases [6-8]. Early $T_{1}$-measurements in liquid $\mathrm{H}_{2} \mathrm{~S}$ have been reported by Hennel et al. $[9,10]$ at saturation pressure $p_{\mathrm{s}}$ up to the critical point in $\mathrm{H}_{2} \mathrm{~S}$. Recently Topol et al. $[11,12]$ presented $T_{1}$-measurements in $\mathrm{H}_{2} \mathrm{~S}$ along the coexistence line from the melting point to temperatures above the critical temperature $T_{\mathrm{c}}$ and found an anomalous temperature and frequency dependence of proton- $T_{1}$ near but above the critical temperature. The only $T_{1-}$ measurements in $D_{2} S$ have been reported by $O$ 'Reilly and Eraker [13] in the liquid phase between the melting point and the boiling point at atmospheric pressure and in the solid phases. No high pressure $T_{1}$-measurements have, to the best of our knowledge, been reported for $\mathrm{H}_{2} \mathrm{~S}$ or $\mathrm{D}_{2} \mathrm{~S}$. 


\section{Experimental}

The deuteron and proton longitudinal relaxation timcs $T_{1}$ were obtained on a Varian XL100-15 FT NMR spectrometer interfaced to a $16 \mathrm{~K}$ Varian $620 \mathrm{~L}-100$ computer with disk accessory by a $t_{1}-90^{\circ}-t_{2}-180^{\circ}-t_{1}-90^{\circ}$ pulse sequence. The observe frequency on this instrument is $100.1 \mathrm{MHz}$ for protons and 15.4 $\mathrm{MHz}$ for deuterons. The modified variable temperature accessory of this spectrometer was used in the experiments. The temperatures were determined to $\pm 0.5 \mathrm{~K}$ with a metal sheathed miniature chromel-alumel thermocouple. Deuterium sulfide was prepared by hydrolysis of degassed aluminium sulfide with $100 \%$ deuterium oxide [14], the isotopic purity as judged from the residual proton peak was $>95 \%$. Hydrogen sulfide was drawn from a lecture bottle. Both sulfides were distilled several times at low temperatures in a high vacuum apparatus. The gaseous sulfides were stored in the dark in a flask over phosphorus pentoxide. Prior to use, the sample was thoroughly degassed by at least five freeze-pump-thaw cycles to a final pressure of $7 \times i 0^{-3} \mathrm{~Pa}$. Freezing was accomplished by immersing the probe into liquid nitrogen, the melting was done in acetone/carbon dioxide mixtures. The high pressure apparatus [15] and the elaborate filling procedure and apparatus [16] necessary for the oxygen-free filling of the high pressure capillaries have been described previously. The purity of the high pressure samples was controlled by comparison of the $T_{1}$-results obtained at saturation pressure with samples of $\mathrm{H}_{2} \mathrm{~S}$ and $\mathrm{D}_{2} \mathrm{~S}$ sealed in heavy walled $5 \mathrm{~mm}$ NMR tubes. No significant difference was found between the two sets of data. As controlled by repeated $T_{1}$-measurements under identical conditions, the sulfides in properly filled and assembled cells remain free of any contamination for several months. In the case of $\mathrm{H}_{2} \mathrm{~S}$ the proton $T_{1}$-data at $p_{\mathrm{s}}$ are also compared to published results $[9,10]$.

The measurements are judged reliable to $\doteqdot 10 \%$, the reproducibility of the data was better than $\pm 5 \%$.

\section{Theoretical}

The dominant relaxation mechanisms for proton and deutcron relaxation in $\mathrm{H}_{2} \mathrm{~S}$ and $\mathrm{D}_{2} \mathrm{~S}$ are the direct dipole-dipole interaction, spin-rotation interaction and quadrupole interaction. The dipole-dipole interaction can be split into an intramolecular and an intermolecular contribution. In the case of proton $T_{1}$ the measured relaxation rate is given by

$\frac{1}{T_{1}}=\frac{1}{T_{1}^{\text {intra }}}+\frac{1}{T_{1}^{\text {inter }}}+\frac{1}{T_{1}^{\text {SR }}}$

whereas the deuterium relaxation rate is given by

$\frac{1}{T_{1}}=\frac{1}{T_{1}^{\mathrm{Q}}}+\frac{1}{T_{1}^{\mathrm{SR}}}$.

In the fast motional limit [17] the dipolar and quadrupoiar relaxation rates are given as [18-20]

$\frac{1}{T_{1}^{\text {intra }}}=\frac{3}{2} \frac{\gamma^{4} \hbar^{2}}{r_{\mathrm{HH}}^{6}} \tau_{2}$

$\frac{1}{T_{1}^{\text {inter }}}=\frac{15}{4} \gamma^{4} h^{2} f_{r} \frac{\rho}{D} \int_{0}^{\infty} \mathrm{d} k\left[\int_{0}^{\infty} \mathrm{d} r g_{\mathrm{HH}}(r) \frac{j_{z}(k r)}{r}\right]^{2}$,

$\frac{1}{T_{1}^{\mathrm{O}}}=\frac{3 \pi^{2}}{2}\left(\frac{e^{2} q Q}{h}\right)^{2}\left(1+\frac{\eta^{2}}{3}\right) \tau_{2}$

with

$\tau_{2}=\int_{0}^{\infty} \mathrm{d} t \frac{\left\langle\mathscr{D}_{m n}^{(2)} \mid \mathscr{D}_{m n}^{(2)}(t)\right\rangle}{\left\langle\left[\mathscr{D}_{m n}^{(2)}\right]^{2}\right\rangle}$,

and $\mathscr{D}$ a Wigner rotation matrix describing the orientation of a molecule fixed coordinate system relative to the laboratory system, $r_{\mathrm{HH}}$ is the proton-proton distance in the molecule, $\rho$ is the density of the system, $D$ is the self-diffusion coefficient, $f_{n}$ is the fractional abundance of the magnetic nuclear species, $j_{z}(k r)$ is a Bessel-function, $g_{\mathrm{HH}}(r)$ the static pair distribution function, $e^{2} q Q / h$ is the quadrupole coupling constant and $\eta_{0}$ the asymmetry parameter of the traceless field gradient tensor. 
Theoretical expressions for the spin-rotation relaxation rate applicable to an asymmetric top are available in the literature within the rotation diffusion model only [21], which, as will be discussed later, seems not to be applicable in the case of $\mathrm{H}_{2} \mathrm{~S}$ and $\mathrm{D}_{2} \mathrm{~S}$. For a spherical top the relaxation rate is given as [22-24]

$\frac{1}{T_{1}^{\mathrm{SR}}}=\frac{8 \pi^{2} I k_{\mathrm{B}} T}{\hbar^{2}}\left(C_{0}^{2} \tau_{J}+2 \Delta C^{2} \tau_{\mathrm{SR}}\right)$

with $\tau_{I}$ the correlation time for the angular momentum autocorrelation function and $\tau_{S R}$ the correlation time of the angular velocity-orientational product correlation function which characterizes anisotropic spin-rotational interactions,

$C_{0}=\frac{1}{3}\left(C_{x x}+C_{y y}+C_{z z}\right)$

and

$\Delta C=\frac{1}{3}\left[C_{z z}-\frac{1}{2}\left(C_{x x}+C_{y y}\right)\right]$

are combinations of the elements of the spin rotation tensor and $I$ is the mean moment of inertia calculated according to

$I^{-1}=\frac{1}{3}\left(I_{x}^{-1}+I_{y}^{-1}+I_{z}^{-1}\right)$.

Table 1 collects the relevant molecular constants needed in the equations given above.

Table 1

\begin{tabular}{llll}
\hline & $\mathrm{H}_{2} \mathrm{~S}$ & $\mathrm{D}_{2} \mathrm{~S}$ & Ref. \\
\hline$r_{\mathrm{H}-\mathrm{H}}[\AA]$ & $1.883 \pm 0.007$ & & {$[5]$} \\
$e^{2} Q Q / h[\mathrm{kHz}]$ & & 149 & {$[4]$} \\
$\eta_{\mathrm{g}}$ & & 0.197 & \\
$C_{0}^{2}\left[\mathrm{~s}^{-2}\right]$ & $2.94 \times 10^{8}$ & $1.86 \times 10^{6}$ & {$[4,5,38]$} \\
$\Delta C^{2}\left[\mathrm{~s}^{-2}\right]$ & $1.52 \times 10^{5}$ & $8.71 \times 10^{3}$ & {$[4,5,38]$} \\
$\langle I\rangle\left[\mathrm{kg} \mathrm{m}^{2}\right]$ & $3.48 \times 10^{-47}$ & $6.75 \times 10^{-47}$ & {$[5]$}
\end{tabular}

The elements of the spin-rotation tensor $\approx$ for $\mathrm{H}_{2} \mathrm{~S}$ have been calculated with the relation $C_{\mathrm{z}}{ }^{*}=\mathrm{g}_{I} \Lambda_{\mathrm{sx}} \cdot B_{\mathrm{z}}$ and $\Lambda_{\mathrm{gx}}$. as given in ref. [4] and the rotational constants $B_{g}$ given in ref. [5] and $g_{I}$ the nuclear $g$-factor [38]. The elements of the moment of inertia tensor I have been calculated from the rotational constants $B_{\varepsilon}(v=0)$ given in ref. [5] and the relation $I_{g}=h / 8 \pi^{2} B_{g}\langle I\rangle$ has been calculated with the relation, $1 /\langle I\rangle=\frac{1}{3} \sum_{g-a, b, e}\left(1 / I_{g}\right)$.

\section{Results and discussion}

The spin-lattice relaxation times $T_{1}$ of the protons in $\mathrm{H}_{2} \mathrm{~S}$ and the deuterons in $\mathrm{D}_{2} \mathrm{~S}$ have been measured. The temperature has been varied from the melting pressure curve to $395 \mathrm{~K}$ and pressures up to $200 \mathrm{MPa}$ have been applied. The relaxation times measured are collected in tables 2 and 3 , the temperature dependences of the relaxation times in $\mathrm{H}_{2} \mathrm{~S}$ and $\mathrm{D}_{2} \mathrm{~S}$ are shown for saturation pressure and $p=$ $200 \mathrm{MPa}$ in fig. 1 .

\subsection{Separation of different relaxation rates}

Regarding for simplicity $D_{2} S$ as a spherical molecule and inserting the pertinent molecular constants (table 1 ) into the theoretical expressions (5) and (6) the experimental relaxation rate is given by

$$
\begin{aligned}
& 1 / T_{1}=3.33 \times 10^{11}\left[\mathrm{~s}^{-2}\right] \tau_{2}[\mathrm{~s}] \\
& +1.23 \times 10^{7}\left[\mathrm{~K}^{-1} \mathrm{~s}^{-2}\right] T\left[\mathrm{~K} \tau_{\tau_{J}}[\mathrm{~s}]\right. \\
& +1.15 \times 10^{5}\left[\mathrm{~K}^{-1} \mathrm{~s}^{-2}\right] T[\mathrm{~K}]_{\tau_{\mathrm{SR}}}[\mathrm{s}] .
\end{aligned}
$$

Because $\tau_{S R} \leqslant \tau_{J}[23,24]$ and $\Delta C^{2} \& C_{0}^{2}$ this expression can be approximated by

$$
1 / T_{1}=3.33 \times 10^{11} \tau_{2}+1.23 \times 10^{7} \tau_{\tau}
$$

Theoretical models dealing with rotational motions of molecules in fluids [25-28] do show that $\tau_{2}$ and $\tau_{J}$ are not independent, but that $\tau_{2}$ has to be regarded as a function of $\tau_{\mathrm{J}}$. This leaves only one free parameter in eq. (8).

Consequently the temperature and pressure dependence of $T_{1}$ should provide a useful test for different diffusion models, although it is hardly possible to determine experimentally both correlation times independently. An additional constraint on these models is the measurement of different isotopes of the same molecular species since any model capable of describing the temperature and pressure dependence of the deuteron- $T_{1}$ in $D_{2} S$ must also be able to account consistently for the temperature and pressure dependence of the proton $-T_{1}$ in $\mathrm{H}_{2} \mathrm{~S}$. Assuming that the reduced correlation times $\tau^{*}=\tau\left(k_{\mathrm{B}} T\right)^{1 / 2} / I$ are at constant $p, T$ 
Table 2

Experimental spin-lattice relaxation times $\left(T_{1}(\mathrm{~s})\right)$ in $\mathrm{H}_{2} \mathrm{~S}$

\begin{tabular}{llllll}
\hline$T$ (K) & $P$ (bar) & & & & \\
& & & & & \\
\hline 100 & 500 & 1000 & 1500 & 2000
\end{tabular}

$\begin{array}{llll}198 & 22.5 & & \\ 211 & 25.5 & 23 & 20 \\ 219 & & & 21.5\end{array}$

$222 \quad 27.5$

225

227

229

232

236

237

238

243

247

252

255

260

266

267

269

274

277

280

286

292

302

303

305

310

321

340

341

393

saturation pressure

\begin{tabular}{|c|c|c|c|c|c|c|c|c|}
\hline$T^{*}(\mathrm{~K})$ & 198 & 208 & 222 & 236 & 247 & 260 & 279 & 308 \\
\hline$T_{1}(\mathrm{~s})$ & 22 & 25.5 & 28 & 28 & 28.5 & 28 & 26.5 & 24.5 \\
\hline $393 \mathrm{~K}:$ & $p(\mathrm{~b}$ & & 100 & 150 & 200 & 250 & & \\
\hline$T_{1}(\mathrm{~s})$ & & & 5 & 12 & 14 & 15 & & \\
\hline
\end{tabular}

identical for both substances yields after insertion of the appropriate molecular constants in eqs. (3), (5) and (6) the following relations:

$\frac{1}{T_{1}^{\mathrm{Q}}}\left(\mathrm{D}_{2} \mathrm{~S}\right)=24.11 \frac{1}{T_{1}^{\text {intra }}}\left(\mathrm{H}_{2} \mathrm{~S}\right)$,
Table 3

Experimental deuterium spin-lattice relaxation times $\left(T_{1}(\mathrm{~s})\right)$ in $\mathrm{D}_{2} \mathrm{~S}$

\begin{tabular}{|c|c|c|c|c|c|c|c|c|}
\hline \multirow[t]{2}{*}{$T(\mathrm{~K})$} & \multicolumn{8}{|c|}{$P$ (bar) } \\
\hline & \multicolumn{2}{|c|}{100} & 500 & \multicolumn{2}{|c|}{1000} & 1500 & \multicolumn{2}{|r|}{2000} \\
\hline 204 & \multicolumn{2}{|c|}{20.5} & & \multirow{2}{*}{\multicolumn{2}{|c|}{20.5}} & & & \\
\hline $\begin{array}{l}220 \\
226\end{array}$ & \multicolumn{2}{|c|}{24.5} & 22.5 & & & & & \\
\hline 230 & & & & & 22 & \multirow{2}{*}{\multicolumn{2}{|c|}{22}} \\
\hline 236 & & & & & & & & \\
\hline 237 & & & 25 & & & & & \\
\hline 238 & \multirow{2}{*}{\multicolumn{2}{|c|}{28.5}} & & \multicolumn{2}{|l|}{24} & & & \\
\hline 250 & & & & \multicolumn{2}{|c|}{25.5} & & & \\
\hline 251 & & 26.5 & & & 25 & & \\
\hline 258 & & & & & & & & 25 \\
\hline 271 & & & & 28 & & & & \\
\hline 272 & 3 & & & & & & & \\
\hline 273 & & & 29.5 & & & & & \\
\hline 275 & & & & & & 29 & & \\
\hline 278 & & & & & & & & 27 \\
\hline 293 & 3 & & 32 & & & & & \\
\hline 294 & & & & 32 & & 31 & & \\
\hline 295 & & & & & & & & 30 \\
\hline 309 & 3 & & 35 & & & & & \\
\hline 324 & & & & & & & & 32 \\
\hline 326 & 3 & & 37 & 36 & & 34 & & \\
\hline 359 & & & & & & 37 & & 37 \\
\hline 360 & 3 & & 39 & 38 & & & & \\
\hline 394 & & & 39 & 40 & & 39 & & 40 \\
\hline 396 & 3 & & & & & & & \\
\hline Saturati & & essure & & & & & & \\
\hline$T(\mathrm{~K})$ & 93 & 208 & 223 & 240 & 257 & 273 & 291 & 307 \\
\hline$T_{1}(\mathrm{~s})$ & 18 & 22 & 24 & 26.5 & 29 & 31 & 34 & 36 \\
\hline
\end{tabular}

$\frac{1}{T_{1}^{\mathrm{SR}}}\left(\mathrm{D}_{2} \mathrm{~S}\right)=1.73 \times 10^{-2} \frac{1}{T_{1}^{\mathrm{SR}}}\left(\mathrm{H}_{2} \mathrm{~S}\right)$.

After separation of the contributions from the intramolecular quadrupole and spin-rotation relaxation mechanism to the experimental relaxation rate in $D_{2} S$ the intramolecular dipole-dipole relaxation rate as well as the spinrotation contribution are obtained for $\mathrm{H}_{2} \mathrm{~S}$ from eqs. (9) and (10). The relaxation rates for all three mechanisms contributing to the measured relaxation rate in the case of $\mathrm{H}_{2} \mathrm{~S}$ can therefore be calculated with eqs. (1), (9) and (10). 

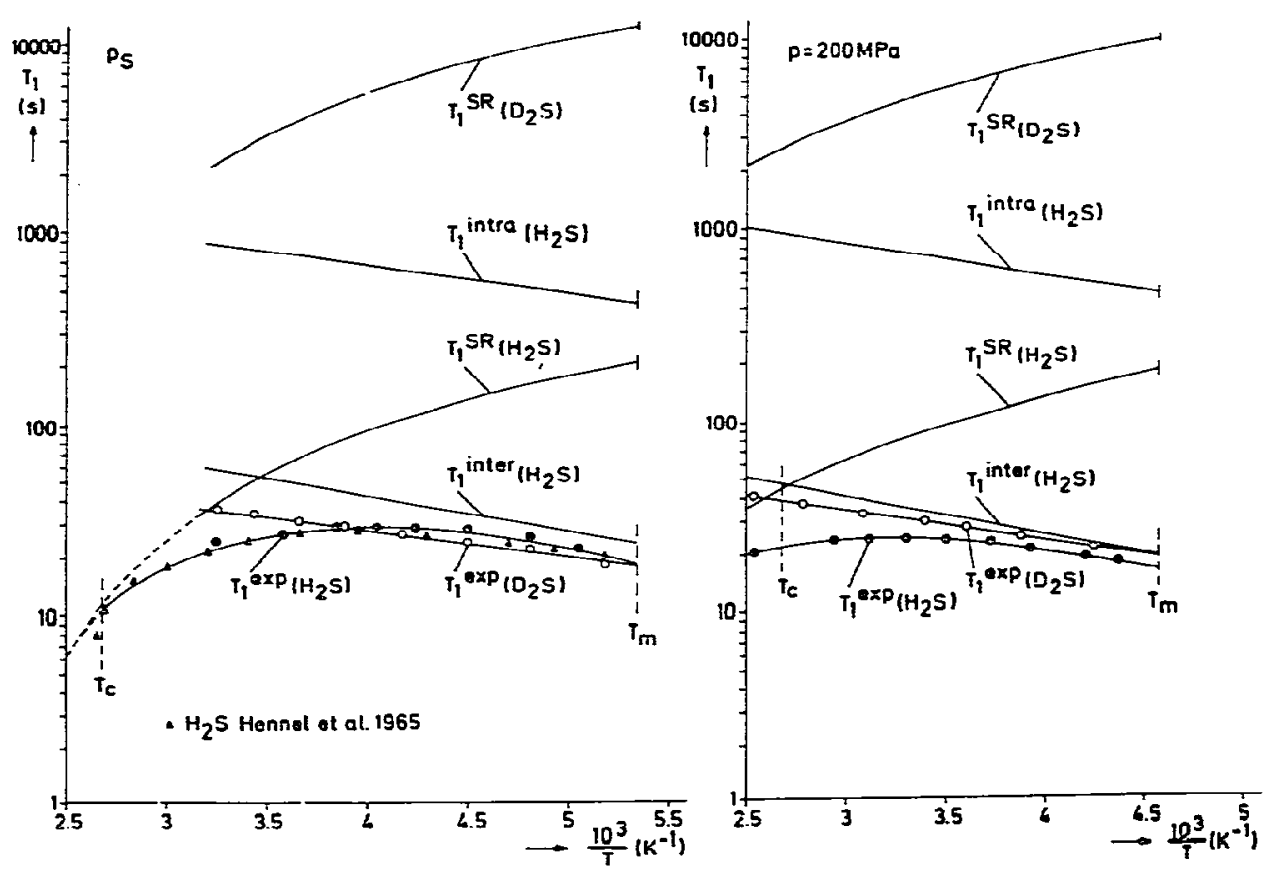

Fig. 1. Temperature dependence of the experimental relaxation times $T_{1}$ in $\mathrm{H}_{2} \mathrm{~S}(-\bigcirc-)$ and $\mathrm{D}_{2} \mathrm{~S}\left(-\mathrm{O}_{-}\right)$together with the estimated contributions from the different relaxation mechanisms for saturation pressure $p_{3}$ and $p=200 \mathrm{MPa}$. $T_{1}$-data for $\mathrm{H}_{2} \mathrm{~S}$ at $p_{\mathrm{s}}$ from refs. $[9,10]$ are included for comparison.

In order to separate with eq. (8) the relaxation rates in the case of $\mathrm{D}_{2} \mathrm{~S}$ a model for the molecular rotational motion must be chosen to provide the functional relationship between $\tau_{2}$ and $\tau_{J}$. The extended $J$-diffusion (EDJ) model $[22,29]$, the Fokker-Planck-Langevin (FPL) model [23] and the rotational diffusion (RD) model $[19,27]$ have been tested. Among these models the EDJ-model only permits the description of all different isobars with a physically acceptable temperature dependence of $\left(T_{1}^{\mathrm{Q}}\right)^{-1}$ and $\left(T_{1}^{\mathrm{SR}}\right)^{-1}$. The necessary parameters $\tau_{J}^{*}$ to fit the $\mathrm{D}_{2} \mathrm{~S}$ data with eq. (8) are compiled in table 4 . The related quantities $\tau_{2}^{*}$ can either be calculated with an equation given by McClung [22] or simply taken from a graph $\tau_{2}^{*}\left(\tau_{3}^{*}\right)$ drawn with the values tabulated by Powles and Rickayzen [26]. It should be noted that even at $T \approx T_{\mathrm{m}}$ the parameters $\tau_{2}^{*}, \tau_{J}^{*}$ necessary to fit the data are beyond the range where all models merge into the rotational diffusion limit, that is they do not obey the Hubbard relation [30].

In the case of both the FPL- and the RDmodel large changes in the parameter $\tau_{\dot{J}}^{\dot{*}}$ in the region of the maximum of the $T_{1}$-isobars are necessary to fit the measured temperature dependence of $T_{1}$ in $D_{2} S$ with eq. (8). Moreover the resulting $T_{1}^{\mathrm{SR}}\left(\mathrm{H}_{2} \mathrm{~S}\right)$ estimated with eq. (10) became shorter than the experimental relaxation times for $\mathrm{H}_{2} \mathrm{~S}$ which is unacceptable.

Although the temperature dependence of $T_{1}$ in $\mathrm{D}_{2} \mathrm{~S}$ can be described quantitatively with the EDJ-model, the resulting relaxation times $T_{1}^{\mathrm{Q}}$ are close to the experimentally determined $T_{I}$ even on the high temperature side of the maximum of the isobars. This behaviour may be due to a deficiency of the separation procedure but every relaxation rate $1 / T_{1}^{\mathrm{SR}}$ large enough to remove the minimum in the $1 / T_{1}^{Q}$-isobars of 
Table 4

\begin{tabular}{|c|c|c|c|c|c|c|c|c|c|c|c|}
\hline \multicolumn{2}{|l|}{$p=p_{\mathrm{s}}$} & \multicolumn{2}{|c|}{$p=10 \mathrm{MPa}$} & \multicolumn{2}{|c|}{$p=50 \mathrm{MPa}$} & \multicolumn{2}{|c|}{$p=100 \mathrm{MPa}$} & \multicolumn{2}{|c|}{$p=150 \mathrm{MPa}$} & \multicolumn{2}{|c|}{$p=200 \mathrm{MPa}$} \\
\hline$T(\mathrm{~K})$ & $\tau \vec{j}$ & $T(\mathrm{~K})$ & 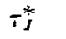 & $T(\mathrm{~K})$ & -5 & $T(\mathrm{~K})$ & $7 \dot{\mathbf{j}}$ & $I(\mathrm{~K})$ & $-j$ & $T(\mathrm{~K})$ & $\tau \xi^{*}$ \\
\hline 193 & 0.25 & 204 & 0.27 & 220 & 0.29 & 220 & 0.26 & \multirow{2}{*}{230} & \multirow{2}{*}{0.275} & & \\
\hline 208 & 0.29 & 226 & 0.35 & 237 & 0.34 & 238 & 0.32 & & & 236 & 0.265 \\
\hline 223 & 0.34 & 250 & 0.44 & 251 & 0.37 & 250 & 0.34 & 251 & 0.33 & 258 & 0.32 \\
\hline 240 & 0.40 & 272 & 0.54 & 273 & 0.44 & 271 & 0.39 & 275 & 0.40 & 278 & 0.37 \\
\hline 257 & 0.47 & 293 & 0.62 & 293 & 0.53 & 294 & 0.50 & 294 & 0.46 & 295 & 0.42 \\
\hline 273 & 0.55 & 309 & 0.80 & 309 & 0.64 & & & & & & \\
\hline 291 & 0.65 & 326 & 0.95 & 326 & 0.75 & 326 & 0.63 & 326 & 0.56 & 324 & 0.48 \\
\hline \multirow[t]{2}{*}{307} & 0.80 & 360 & 1.50 & 360 & 1.00 & 360 & 0.84 & 359 & 0.69 & 359 & 0.59 \\
\hline & & 396 & 2.25 & 394 & 1.35 & 394 & 1.05 & 394 & 0.93 & 394 & 0.77 \\
\hline
\end{tabular}

$\mathrm{D}_{2} \mathrm{~S}$ proved, after transformation with eq. (10), to be larger than $1 / T_{1}^{\text {exf }}$ for $\mathrm{H}_{2} \mathrm{~S}$, which of course is physically impossible. It is to be expected that the relaxation time $T_{1}$ in $D_{2} S$ possesses a weak anomaly in the vicinity of the critical temperature similar to that found in the case of $\mathrm{H}_{2} \mathrm{~S}$ by Topol et al. $[11,12]$. These authors measured a sharp rise in the proton-relaxation rate $1 / T_{1}$ in $\mathrm{H}_{2} \mathrm{~S}$ near bu: above the critical temperature. However, our experimental equipment does not permit sufficiently precise measurements around $T_{\mathrm{c}}$ to resolve this question. Thus in the subsequent discussion we will focus our attention on the behaviour of the relaxation times $T_{1}$ at temperatures $T<T_{c}$.

The isobaric temperature dependence of the relaxation times corresponding to the different relaxation mechanisms in $\mathrm{D}_{2} \mathrm{~S}$ and $\mathrm{H}_{2} \mathrm{~S}$ are shown in fig. 1 for $p=p_{s}$ and $p=200 \mathrm{MPa}$ only. They cannot be expected to be very precise since in view of the difference in the relative strength of the relaxation mechanisms operative in $\mathrm{D}_{2} \mathrm{~S}\left(3.33 \times 10^{11} \gg 1.23 \times 10^{\top} \mathrm{T}\right)$ the $T_{1}^{\mathrm{SR}}$ estimated may contain a relatively large error. The separation procedure presented here obviously cannot provide a critical test of the different diffusional models considered. Not withstanding these difficulties in the case of $\mathrm{D}_{2} \mathrm{~S}$ the experinental relaxation rates are almost entirely due to quadrupolar relaxation so that $T_{1}^{\mathrm{O}}$ is not included in fig. 1 . The spin-rotational contribution as obtained with the EDJ-model (in fact every model should lead to analogous results in this respect) is completely negligible due to the smallness of the components of the spin-rotation coupling tensor $\mathbf{c}$. As can be seen from fig. 1, this does not hold for the protons of hydrogen sulfide. The spin-rotational relaxation in $\mathrm{H}_{2} \mathrm{~S}$ contributes significantly to the total relaxation rate of the protons and becomes the dominating relaxation mechanism at temperatures near the critical temperature $T_{\mathrm{c}}$. At low temperatures the intermolecular dipolar interaction is the dominating relaxation mechanism, whereas the intramolecular dipolar interaction is the least effective relaxation mechanism at all temperatures and pressures. This is a consequence of the fast rotational motions of the molecules in liquid $\mathrm{H}_{2} \mathrm{~S}$ and $\mathrm{D}_{2} \mathrm{~S}$ which result in very short effective correlation times $\tau_{2}$. The relative magnitude of inter- and intramolecular dipolar relaxation rates in hydrogen sulfide contrasts markedly with the behaviour of these quantities in liquid ammonia and liquid water as can be illustrated by the ratio of these relaxation rates in these systems at their respective melting points for $p=p_{\mathrm{s}}$

$$
\begin{array}{rlrl}
R_{1}^{\text {inter }} / R_{1}^{\text {intra }} & =0.5 & & \text { for } \mathrm{H}_{2} \mathrm{O} \\
& \approx 18 & & \text { for } \mathrm{H}_{2} \mathrm{~S} \\
& \approx 1.8 & \text { for } \mathrm{NH}_{3} .
\end{array}
$$

However a concise discussion of the intermolecular relaxation rate in these systems in terms of eq. (4) must await a thorough experimental determination of the constituent quantities in this expression especially in the case of hydrogen sulfide. 
A discussion of the results presented here in terms of the density dependence of the parameters involved in the various models [31] is not possible at present due to the lack of experimental densities for $\mathrm{H}_{2} \mathrm{~S}$ and $\mathrm{D}_{2} \mathrm{~S}$ at pressures substantially higher than $p_{\mathrm{s}}$. For example fig. 2 shows that the density dependence of the parameter $(\tau \dot{\bar{j}})^{-1}$ at saturation pressure [37] follows closely the density dependence of $\left(\tau_{\omega}^{*}\right)^{-1}$ calculated within the rough hard sphere model [25] with a hard sphere diameter $\sigma$ between $3.95-4 \AA$. These values are close to the molecular diameters calculated from

$$
\begin{array}{ll}
\text { liquid density at } T=192 \mathrm{~K}: & 2 a=4.34 \AA, \\
\text { viscosity of the gas: } & 2 a=4.32 \AA, \\
\text { van der Waals constant } b: & 2 a=3.24 \AA, \\
\text { crystal structure (solid I): } & 2 a=4.1 \AA .
\end{array}
$$

It is also close to the parameter $u-3.405 \AA$ [32] in the Lennard-Jones potential of argon with which hydrogen sulfide is isoelectronic. Thus it may well be that the rough hard sphere fluid represents a useful approximation to real liquid hydrogen sulfide.

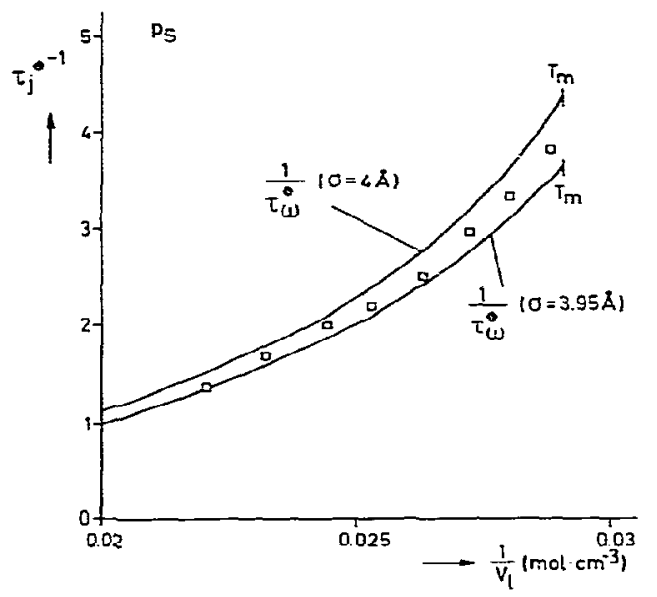

Fig. 2. Density dependence of $\left(\tau_{j}^{*}\right)^{-1}$ for saturation pressure $p_{s}$ and $\left(\tau_{w}^{*}\right)^{-1}$ versus density for the rough hard sphere fluid as calculated with eqs. (3.2)-(3.4) and (3.17) given in ref. [25] for $\sigma=3.95 \AA$ and $\sigma=4 \AA$. The molar volumes of $\mathrm{D}_{2} \mathrm{~S}$ at saturation pressure have been taken from reí. [37].

\subsection{Pressure and temperature dependence of the relaxation times}

As can be seen from fig. 1 the isobaric temperature dependence of $T_{1}^{\mathrm{Q}}, T_{1}^{\text {intra }}$ and $T_{1}^{\text {inter }}$ show simple Arrhenius-behaviour at low temperatures. The slope of the isobars leads to an apparent "activation enthalpy" defined through the relation

$\Delta H^{\dot{*}}=\left.R \frac{\partial \ln \left(1 / T_{\mathrm{s}}\right)}{\partial(1 / T)}\right|_{p}$,

which amounts to

$$
\begin{aligned}
& T_{1}^{\mathrm{Q}}, T_{1}^{\mathrm{intra}}: \Delta H^{\ddagger}=3 \pm 0.2[\mathrm{~kJ} / \mathrm{mol}], \\
& T_{1}^{\text {inter }}: \Delta H^{\ddagger}=4 \pm 0.2[\mathrm{~kJ} / \mathrm{mol}] .
\end{aligned}
$$

This has to be compared with the mean thermal energy at $T=395 \mathrm{~K}: K T=3.3[\mathrm{~kJ} / \mathrm{mol}]$ and at $T=200 \mathrm{~K}: R T \approx 1.66[\mathrm{~kJ} / \mathrm{mol}]$. The comparison shows that the description of the molecular motions in liquid hydrogen sulfide as activated processes is not applicable. O'Reilly et al. [13] obtained an activation energy of $E_{\mathrm{A}}=$ $3.7[\mathrm{~kJ} / \mathrm{mol}]$ for ${ }^{2} H-T_{1}$ in solid $D_{2} S$. Thus rotational motions of the molecules are only slightly more hindered in the solid I phase than in the liquid phase. From neutron scattering data in liquid $\mathrm{H}_{2} \mathrm{~S}$ Rzany and Sciesimski [3] estimated an interaction energy of $E=1.9[\mathrm{~kJ} / \mathrm{mol}]$ due to permanent dipole interactions. Thus these interactions contribute significantly to the hindrance of rotational motions of $\mathrm{H}_{2} \mathrm{~S}$ molecules in the liquid. As the permanent dipole moments of $\mathrm{H}_{2} \mathrm{O}$ and $\mathrm{NH}_{3}$ are not much different from that of $\mathrm{H}_{2} \mathrm{~S}$, comparison of the barriers to rotational motions in these systems indicates the dominating influence of hydioger bonding interactions upon these molecular motions. Fig. 3 compares the temperature dependence of ${ }^{2} \mathrm{H} T_{1}$ in $\mathrm{D}_{2} \mathrm{~S}$, $\mathrm{ND}_{3}[16]$ and $\mathrm{D}_{2} \mathrm{O}$ [33] at saturation pressure and at $p=200 \mathrm{MPa}$. The relaxation times $T_{1}$ are shown in an Arrhenius-plot over the reduced inverse temperature $1 / T_{\mathrm{r}}=T_{\mathrm{c}} / T\left(T_{\mathrm{c}}=\right.$ critical temperature). Obviously the influence of hydrogen bonding interactions upon molecular motions in the associated dipolar fluids $\mathrm{ND}_{3}$ and $\mathrm{D}_{2} \mathrm{O}$ lead to significantly higher apparent activation enthalpies. Whereas in liquid $\mathrm{D}_{2} \mathrm{~S}$ and $\mathrm{ND}_{3}$ 


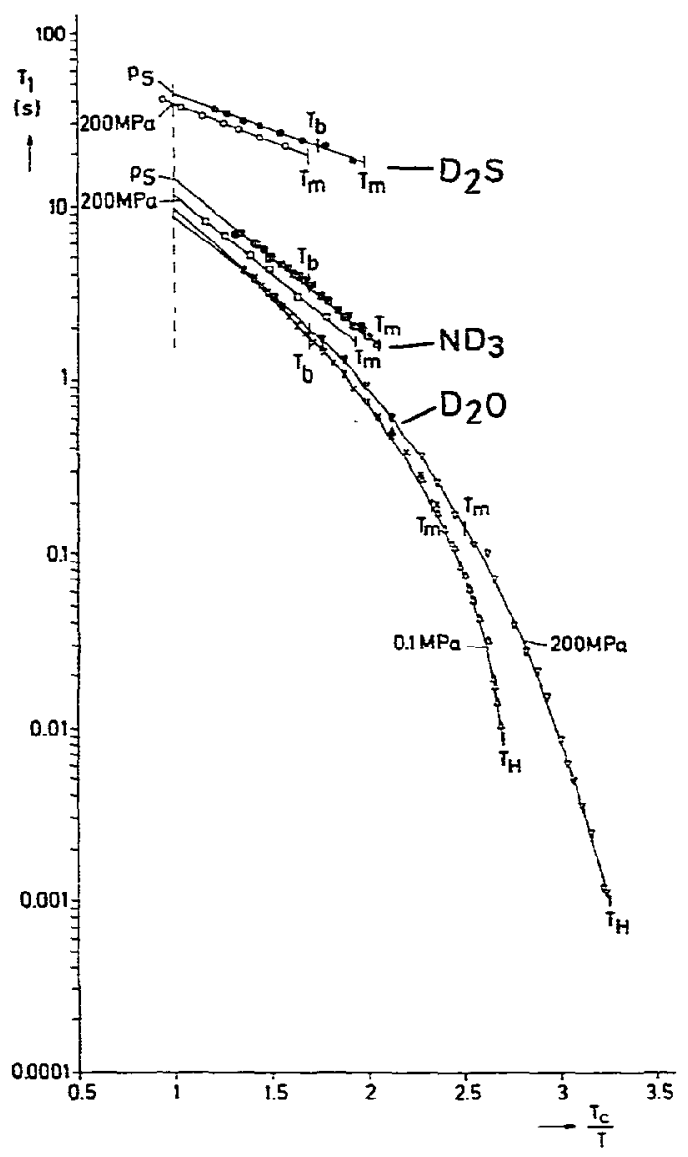

Fig. 3. $T_{1}\left({ }^{2} H\right)$ for $D_{2} O, D_{2} S$ and $N D_{3}$ versus reduced inverse temperature $1 / T_{\mathrm{r}}=T_{c} / T . T_{\mathrm{m}}$-melting point temperature, $T_{b}$-boiling point temperature under atmospheric pressure, $T_{\mathrm{H}}$-homogeneous nucleation temperature [1], $\Delta$, $\nabla$ data from ref. [33], $\Delta, \nabla$ data from ref. [39], $\times$ data calculated with a two exponential fit equation given in ref. [40], $\square$, 圈 data from ref. [16].

[16] this parameter is virtually constant over the whole range of temperatures and pressures measured, it becomes temperature- and pressure-dependent in the hydrogen bonded network-liquid water. Furthermore comparison of the reduced correlation times $\tau_{2}^{*}$ should refiect also the influence of hydrogen bonds upon molecular mobility:

\begin{tabular}{lllll} 
& $\mathrm{D}_{2} \mathrm{O}$ & $\mathrm{D}_{2} \mathrm{~S}$ & \multicolumn{2}{l}{$\mathrm{ND}_{3}$} \\
\hline$\tau_{2}^{*}$ & 86 & 1.03 & 6.5 & $T=T_{\mathrm{m}}, p=p_{\mathrm{s}}$ \\
& 12 & 0.89 & 3.8 & $T_{\mathrm{r}}=0.56, p=p_{\mathrm{s}}$
\end{tabular}

Obviously the effective time constant for orientational fluctuations in hydrogen sulfide is very short, which shows again that the reorientational motions are only slightly hindered, whereas hydrogen bonds block these motions appreciably in the associated liquids especially in the network-liquid water. Regarding the pressure dependence of the relaxation times one can define an "activation volume" $\Delta V^{\ddagger}$ through the relation:

$$
\Delta V^{\ddagger}=\left.R T \frac{\partial \ln \left(1 / T_{1}\right)}{\partial p}\right|_{\tau}
$$

At temperatures $T \leqslant 325[\mathrm{~K}] \Delta V^{*}$ is within experimental error independent of pressure for both $1 / T_{1}^{Q}, 1 / T_{1}^{\text {inura }}$ (fig. 4) and $1 / T_{1}^{\text {inter }}$ (fig. 5) and at $T=300 \mathrm{~K}\left(T_{\mathrm{r}}=0.8\right)$ amounts to $\Delta V_{\text {intra }}^{\ddagger}=2 \pm 0.5\left[\mathrm{~cm}^{3} / \mathrm{mol}\right]$ and $\Delta V_{\text {inter }}^{\ddagger}=$ $7 \pm 1\left[\mathrm{~cm}^{3} / \mathrm{mol}\right]$. Comparison with $\Delta V^{\ddagger}$ obtained in liquid ammonia [16] shows that in both liquids $\Delta V_{\text {intra }}^{\ddagger}$ is nearly equal at the same reduced temperatures, but that $\Delta V_{\text {inter }}^{+}$is somewhat higher in hydrogen sulfide than in

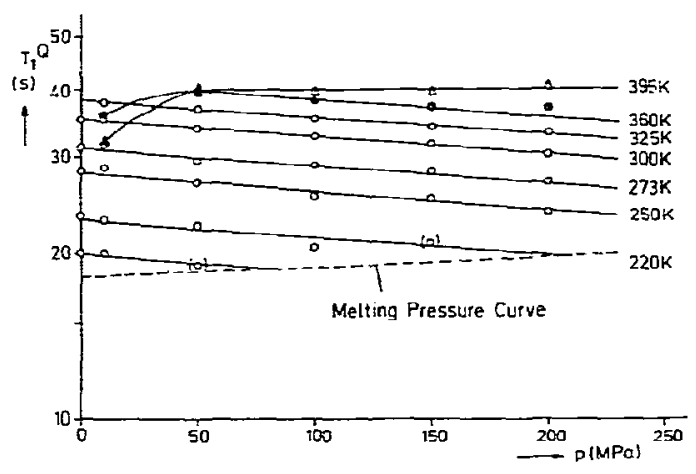

Fig. 4. Isotherms of the relaxation times $T_{1}^{\circ}$ of the intramolecular quadrupole relaxation mechanism in $\mathrm{D}_{2} S$. The corresponding relaxation time $T_{1}^{\text {intra }}$ of the intramolecular dipole-dipole relaxation mechanism possesses an identical pressure dependence according to eq. (9). 


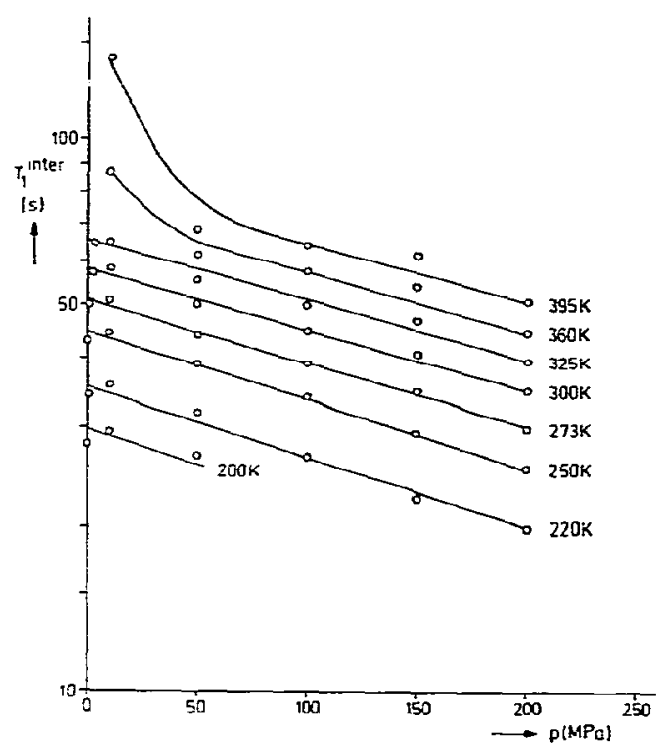

Fig- 5. Isotherms of the relaxation times $T_{1}^{\text {inter }}$ of the intermolecular dipole-dipole relaxation mechanism in $\mathrm{H}_{2} \mathrm{~S}$.

liquid ammonia. However, in both liquids the pressure dependence of the translational motions, as reflected in the parameter $\Delta V_{\text {inter, }}^{\ddagger}$ is larger than the corresponding pressure dependence for the reorientational motions. $\Delta V^{\ddagger}$ may be regarded as a qualitative measure of the space required by a molecule to reorient or translate to a new position in the cage of its next neighbours. Thus $\Delta V_{\text {inter }}^{i}>\Delta V_{\text {intra }}^{\frac{F}{i}}$ indicates that translational diffusion is slowed down faster with increasing density than is rotational diffusion.

\section{Conclusions}

The spin-lattice relaxation times in liquid $\mathrm{H}_{2} \mathrm{~S}$ and $D_{2} S$ have been measured since its molecular geometry and molecular parameters (table 1) are similar to those of the water molecule but its intermolecular interactions are not dominated by hydrogen bonding interactions. Thus a study of its dynamic behaviour as a function of temperature and pressure should help to understand the contribution of hydrogen bonds to the hindrance of rotational and translational motions of the molecules in the liquid phase. The effective corrclation timcs of orientational fluctuations in $\mathrm{H}_{2} \mathrm{~S}$ and $\mathrm{D}_{2} \mathrm{~S}$ are very short $\left(\tau_{2}=10^{-14} \mathrm{~s}\right)$. The reduced effective time constants $\tau_{2}^{*}$, which measure the correlation times of the fiuctuations relative to those of a hypothetical free rotor, obey at all temperatures and pressures measured the relation $\tau_{i}^{*} \leqslant 1$. This shows that rotational motions in this liquid must proceed rather freely. The corresponding correlation times in liquid ammonia [16] are an order of magnitude longer and in liquid water they increase for identical reduced conditions by more than two orders of magnitude $[33,34]$. Furthermore, fig. 3 shows a much stronger isobaric temperature dependence of the correlation times in the associated liquids $\mathrm{ND}_{3}$ and $\mathrm{D}_{2} \mathrm{O}$ than in the non-associated liquid $\mathrm{D}_{2} \mathrm{~S}$. A comparison of the "activation enthalpies" obtained for rotational and translational motions in $\mathrm{H}_{2} \mathrm{~S}, \mathrm{H}_{2} \mathrm{O}$ [34] and $\mathrm{NH}_{3}$ [16] reveals, that the concept of an activated process is certainly not applicable to transport phenomena in liquid $\mathrm{H}_{2} \mathrm{~S}$, since the barrier to rotations is comparable to the mean thermal energy.

Unfortunately due to the lack of mcasured densities at pressures $p>p_{\mathrm{s}}$ a more thorough discussion of the density dependence of the correlation times $\tau_{2}$ and $\tau_{j}[25,31,35,36]$ was not possible. The finding, that the data could be described with the EDJ-modei does not present a critical test of this model because the data treatment had to rely on the estimation of the spin-rotational relaxation rate in $\mathrm{D}_{\mathbf{2}} \mathrm{S}$ where it makes only a minor contribution to the total relaxation rate. However, the condition that any model must describe the pressure and temperature dependence of the relaxation times in $\mathrm{D}_{2} \mathrm{~S}$ as well as in $\mathrm{H}_{2} \mathrm{~S}$ consistently led to the exclusion of the FPL- and the RD-mode!. This may however be due to the unavailability of a theoretical treatment of rotational motions for an asymmetric top which hydrogen sulfide in fact is. Some of the difficulties of the present separation procedure would be removed if self- 
diffusion coefficients and static proton-proton pair-correlation functions or shear viscosities would be available over the range of temperatures and pressures mcasurcd. This would allow one to estimate, at least approximately, the intermolecular dipolar relaxation rate and thus provide an alternative separation of the different relaxation rates. Furthermore a comparison of the influence of the structure of liquid hydrogen sulfide, liquid ammonia and liquid water in terms of eq. (4) on the dynamical behaviour of the molecules in these liquids. would become possible.

The high temperature maximum of ${ }^{2} \mathrm{H} T_{1}$ in $\mathrm{D}_{2} \mathrm{~S}$, its reduction with increasing pressure, and complete disappearence at pressures $p \geqslant$ $200 \mathrm{MPa}$ is interesting since it cannot be explained by a balance of $T_{1}^{\mathrm{SR}}$ and $T_{1}^{\mathrm{Q}}$ in $\mathrm{D}_{2} \mathrm{~S}$. In fact any reasonable estimate of $T_{1}^{\mathrm{SR}}$ fails to remove the maximum in $T_{1}^{\mathrm{Q}}$. This indicates an anomalous temperature dependence of $T_{1}^{\mathrm{O}}$ in $\mathrm{D}_{2} \mathrm{~S}$ at high temperatures and low pressures. Whether this anomaly is analogous to the one found above $T_{\mathrm{c}}$ in $\mathrm{H}_{2} \mathrm{~S}$ [11] deserves further careful measurements at high temperatures.

\section{Acknowledgement}

The expert technical assistance by $\mathrm{Mr}$. R. Knott and Mr. S. Heyn made this study feasible. Their contribution is gratefully acknowledged. The work presented here was supported by the Deutsche Forschungsgemeinschaft and the Fonds der Chemischen Industrie.

\section{References}

[1] C.A. Angell in: Water-a comprehensive treatise, Vol. 7, ed. F. Franks (Plenum Press, New York 1981).

[2] 1.C. Hindman, A. Svirmickas and W.B. Dixon, J. Chem. Phys. 47 (1967) 4658.

[3] H. Rzany and J. Sciesinski, Physica 29 (1963) 488.

[4] F.C. DeLucia and I.W. Cederberg, J. Mol. Spectry. 40 (1971) 52 .

[5] R.L. Cook, F.C. DeLucia and P. Helminger, J. Mol. Struct. 28 (1975) 237.
[6] Z.M. El Saffar and P. Schultz, J. Chem. Phys. 56 . (1971) 2524.

[7] J.H. Loehlin, P.G. Mennitt and J.S. Waugh, J. Chem. Phys. 44 (1966) 3912.

[8] D.C. Look, 1.J. Lowe and J.A. Northby, I. Chem. Phys. 44 (1966) 3441.

[9] J.W. Hennel, A. Jasinski, S. Sagnowski and T. Waluga, Acta Phys. Polon. 28 (1965) 673.

[10] J.W. Hennel and T. Waluga, Bulletin Ampère 10 (1961) 366.

[11] R. Topol, K. Krynicki and J.G. Powles, Mol. Phys. 37 (1979) 1655.

[12] R. Topol, Mol. Phys. 40 (1980) 161.

[13] D.E. O'Reilly and J.H. Eraker, J. Chem. Phys. 52 (1970) 2407.

[14] M. Bandler, in: Handbuch der Präparativen Anorganischen Chemie, ed. G. Brauer (Stuttgart, 1975).

[15] G. Völkel, E. Lang and H.-D. Lüđemann, Ber. Bunsenges. Physik. Chem. 83 (1979) 722.

[16] H. Hauer, E. Lang and H.-D. Lüdemann, Ber. Bunsenges. Physik. Chem. 83 (1979) 1262.

[17] A. Abragam, The principles of nuclear magnetism (Oxford Univ. Press, London, 1961).

[18] M.D. Zeidler, Ber. Bunsenges. Physik. Chem. 75 (1971) 229.

[19] H.W. Spiess, in: NMR-basic principles and progress, Vol. 15, eds. P. Diehl, E. Fluck, R. Kosfeld (Springer, Berlin, 1978).

[20] D. Pumpernick and A. Azman, Chem. Phys. Letters 34 (1975) 438.

[21] C.H. Wang, Mol. Phys. 28 (1974) 801.

[22] R.E.D. McClung, Advan. Mol. Relaxation Inter. Processes 10 (1977) 83.

[23] R.E.D. McClung, J. Chem. Phys. 73 (1980) 2435.

[24] G. Lévi, J.P. Marsault, F. Marsault-Hérail and R.E.D. McClung, J. Chem. Phys. 73 (1980) 2443.

[25] J. O'Dell and B.J. Berne, J. Chem. Phys. 63 (1975) 2376.

[26] J.G. Powles and G. Rickayzen, Mol. Phys. 33 (1977) 1207.

[27] J. McConnell, Rotational brownian motion and dielectric theory (Acadenic Press, London, 1980).

[28] D.E. O'Reilly, J. Chem. Phys. 63 (1973) 3177.

[29] D. Chandler, J. Chem. Phys. 60 (1974) 3508.

[30] P.S. Hubbard, Phys. Rev. 131 (1963) 1155.

[31] J. Jonas, Ann. Rev. Phys. Chem. 26 (1975) 167.

[32] I.R. McDonald and X. Singer, J. Chem. Phys. 50 (1969) 2308.

[33] E. Lang and H.-D. Lüdemann, Ber. Bunsenges. Physik. Chem. 84 (1980) 462.

[34] E. Lang and H.-D. Lüdemann, J. Chem. Phys. 67 (1977) 718 .

[35] W.J. Lamb and J. Jonas, J. Chem. Phys. 74 (1981) 913.

[36] J.J. van Loef, Physica 103B (1981) 133.

[37] E.C.W. Clarke and D.N. Glew, Can. J. Chem. 48 (1970) 764. 
[38] A. Carrington and A.D. McLachlan, Introduction to magnetic resonance (Harper and Row, New York, 1969).
[39] J. Jonas, T. DeFries and D.J. Wilbur, J. Chem. Phys. 65 (1976) 582.

[40] J.C. Hindman, J. Chem. Phys. 60 (1974) 4488. 